\title{
Quantization of setup uncertainties in 3-D dose calculations
}

\author{
Anthony E. Lujan a) and Randall K. Ten Haken \\ Department of Radiation Oncology, University of Michigan, Ann Arbor, Michigan 48109 \\ Edward W. Larsen \\ Department of Nuclear Engineering and Radiological Sciences, University of Michigan, \\ Ann Arbor, Michigan 48109 \\ James M. Balter \\ Department of Radiation Oncology, University of Michigan, Ann Arbor, Michigan 48109
}

(Received 3 December 1998; accepted for publication 3 August 1999)

\begin{abstract}
Random setup errors can lead to erroneous prediction of the dose distribution calculated for a patient using a static computed tomography (CT) model. Multiple recomputations of the dose distribution covering the range of expected patient positions provides a way to estimate a course of treatment. However, due to the statistical nature of the setup uncertainties, many courses of treatment must be simulated to calculate a distribution of average dose values delivered to a patient. Thus, direct simulation methods can be time consuming and may be impractical for routine clinical treatment planning applications. Methods have been proposed to efficiently calculate the distribution of average dose values via a convolution of the dose distribution (calculated on a static CT model) with a probability distribution function (generally Gaussian) that describes the nature of the uncertainty. In this paper, we extend the convolution-based calculation to calculate the standard deviation of potential outcomes $\sigma_{D}(x, y, z)$ about the distribution of average dose values, and we characterize the statistical significance of this quantity using the central limit theorem. For an example treatment plan based on a treatment protocol in use at our institution, we found that there is a $68 \%$ probability that the actual dose delivered to any point $(x, y, z)$ will be within $3 \%$ of the average dose value at that point. The standard deviation also yields confidence limits on the dose distribution, and these may be used to evaluate treatment plan stability. (C) 1999 American Association of Physicists in Medicine. [S0094-2405(99)02411-6]
\end{abstract}

Key words: patient setup uncertainty, treatment verification, treatment planning, dose calculations

\section{INTRODUCTION}

Uncertainties arising from daily setup errors and organ motion can lead to differences between the dose distribution on a treatment plan and the actual dose distribution delivered to a patient. Two primary approaches exist to account for these uncertainties. The traditional approach measures or estimates the extent of setup uncertainty and organ motion and adds margins around a clinical target volume (CTV) to form a planning target volume (PTV). The dose is calculated on a static patient model and prescribed to the PTV, with the intent that the actual dose delivered to the CTV will be equivalent to the predicted dose distribution. This margin expansion approach does not account for the differences between the predicted dose distribution and the actual delivered dose distribution for normal tissues near the CTV. The second approach includes margins for errors and incorporates the uncertainties directly into the dose calculations, thereby giving a more complete and accurate prediction of the delivered dose distribution to both the target volume and normal tissues.

Methods based on a convolution of the static dose distribution with a function (generally Gaussian) representing the distribution of random uncertainties from setup and organ motion have been proposed for sites in the pelvis. ${ }^{1-5}$ In a previous paper, we described a convolution-based method to incorporate uncertainties from intratreatment organ motion due to breathing into 3-D dose calculations. ${ }^{6}$ In the present work, we generalize this method to incorporate uncertainties due to daily setup errors into 3-D dose calculations for radiotherapy. In doing this, we quantitatively describe potential differences between convolution-based predictions of the dose distribution and the actual dose delivered in a finitefractioned course of treatment. We confirm the validity of our approach via comparisons to direct simulations for treatment of tumors in the liver. Also, we retrospectively analyze the effects of these uncertainties on the treatment plan and dose prescriptions based on a treatment protocol for liver disease used at the University of Michigan. ${ }^{7-9}$

\section{METHODS AND MATERIALS}

The basic algorithm for convolving setup uncertainties with a static dose distribution has been described previously. ${ }^{1-5}$ The convolution method assumes rigid body motion, no change in the patient external contour, and no organ deformation. In the present study, we consider random translational setup uncertainties along the anterior-posterior (AP), left-right (LR), and superior-inferior (SI) axes.

Based on a retrospective analysis of our patient setup by Schewe et al. ${ }^{10}$ we assume that the translations along these primary axes are independent and that the nature of the random translational setup uncertainties can be characterized by Gaussian probability distribution functions, as shown in Fig. 


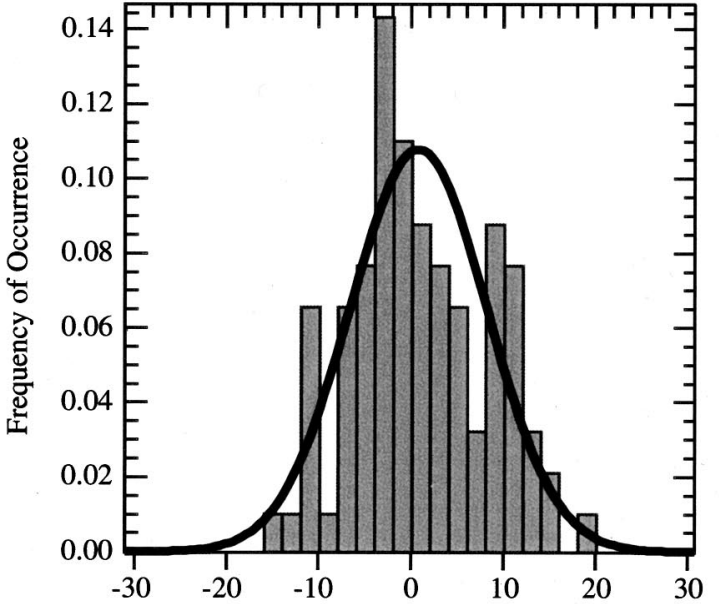

LR Setup Error (mm)

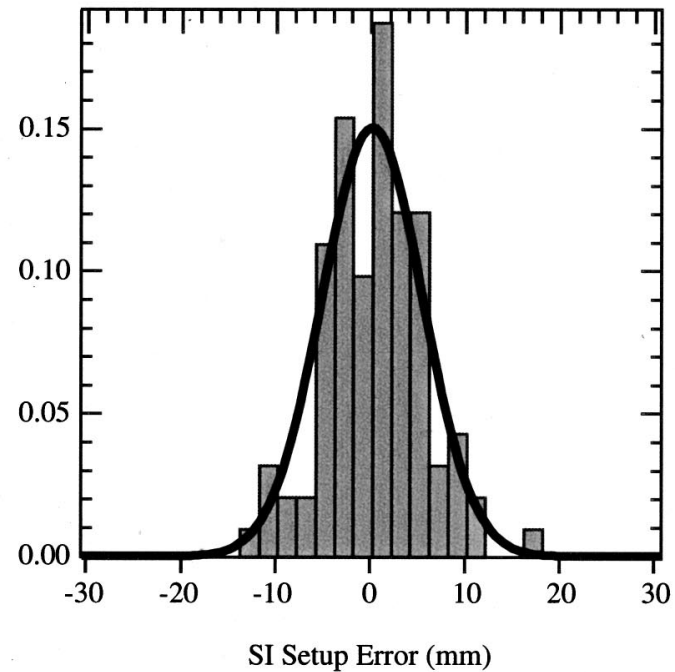

FIG. 1. Measured distribution of setup errors in the LR and SI directions and Gaussian model of data (solid line) for treatments to sites in the abdomen.

1 for treatment to sites in the abdomen. In this figure, the origin of coordinates corresponds to the nominal position of the patient assuming no systematic error (i.e., the static patient position at treatment planning). The mean setup uncertainty is assumed to be zero for all three axes, and we used the standard deviations in translation from Schewe's study ( $\sigma_{\mathrm{LR}}=7.4 \mathrm{~mm}, \sigma_{\mathrm{AP}}=4.9 \mathrm{~mm}, \sigma_{\mathrm{SI}}=5.3 \mathrm{~mm}$ ).

The distribution of average dose values $\bar{D}(x, y, z)$, including random translational setup uncertainties calculated using a convolution-based method, is computed using Eq. (1):

$$
\begin{aligned}
\bar{D}(x, y, z)= & \iiint D_{0}\left(x^{\prime}, y^{\prime}, z^{\prime}\right) \\
& \times N\left(x^{\prime}-x, y^{\prime}-y, z^{\prime}-z\right) d x^{\prime} d y^{\prime} d z^{\prime},
\end{aligned}
$$

where $\bar{D}(x, y, z)$ is the mean dose to any point $x, y, z$ including uncertainties; $D_{0}\left(x^{\prime}, y^{\prime}, z^{\prime}\right)$ is the static dose to a point $x^{\prime}$, $y^{\prime}, z^{\prime} ; N$ is the normalized probability distribution function describing setup uncertainties in 3-D along the LR, AP, and SI axes, respectively; $N=N_{x}\left(x^{\prime}-x\right) N_{y}\left(y^{\prime}-y\right) N_{z}\left(z^{\prime}-z\right)$; and $N_{x}\left(x^{\prime}-x\right)=e^{-\left(x^{\prime}-x\right)^{2} / 2 \sigma_{x}^{2} / \sigma_{x} \sqrt{2 \pi}}$, is the normal distribution with standard deviation $\sigma_{x}$ about a point $x$, similar for $y$ and $z$.

In theory, the integration is carried out over all space $( \pm \infty)$, but for a practical implementation, we cut off the integration at $\pm 3 \sigma$ in each direction and renormalize. As noted by Leong ${ }^{1}$ and Killoran, ${ }^{11}$ the distribution of average dose values calculated using Eq. (1) represents the dose distribution received by the patient given an infinite number of small fractions. However, a real course of treatment is delivered with a finite number of fractions.

Leong proposed characterizing the potential difference between a real finite fractioned treatment and the distribution of average dose values $\bar{D}$ by the standard deviation $\sigma_{D}$ of the average dose distribution. Killoran proposed using a Monte Carlo-based direct simulation method to account for the finite nature of treatment delivery (a course of treatment is simulated multiple times, allowing for the computation of the mean dose distribution as well as a range of possible out- comes about the mean dose distribution). However, direct simulations can be time consuming and often impractical for regular treatment planning applications.

Hence, we extended the convolution-based method to allow for an efficient computation of the standard deviation $\sigma_{D}$ of the average expected dose distribution $\bar{D}$. Our method for calculating $\sigma_{D}$ and the statistical significance of this quantity is given below.

The general expression for the standard deviation ${ }^{12}$ is

$$
\begin{aligned}
\sigma_{D}(x, y, z)= & {\left[\iiint\left[D_{0}\left(x^{\prime}, y^{\prime}, z^{\prime}\right)-\bar{D}(x, y, z)\right]^{2}\right.} \\
& \left.\times N\left(x^{\prime}-x, y^{\prime}-y, z^{\prime}-z\right) d x^{\prime} d y^{\prime} d z^{\prime}\right]^{1 / 2} .
\end{aligned}
$$

Equation (2) can be expanded and expressed as

$$
\begin{aligned}
\sigma_{D}(x, y, z)= & {\left[\left(\iiint D_{0}^{2}\left(x^{\prime}, y^{\prime}, z^{\prime}\right)\right.\right.} \\
& \left.\times N\left(x^{\prime}-x, y^{\prime}-y, z^{\prime}-z\right) d x^{\prime} d y^{\prime} d z^{\prime}\right) \\
& \left.-\left(\bar{D}^{2}(x, y, z)\right)\right]^{1 / 2} .
\end{aligned}
$$

Equation (3) gives the standard deviation that would result from an entire treatment delivered in a single fraction with a setup uncertainty characterized by $N\left(x^{\prime}-x, y^{\prime}-y, z^{\prime}-z\right)$. Real treatments are delivered over $M$ multiple fractions, and so the standard deviation for a fractionated plan is smaller than $\sigma_{D}$ by $1 / \sqrt{M}$. For a fractionated plan, the probability that the absolute difference between a real treatment consisting of $M$ fractions $D_{M}$ and $\bar{D}(x, y, z)$ at any point $x, y, z$ will be less than $\sigma_{D}(x, y, z) / \sqrt{M}$ can be expressed using the central limit theorem ${ }^{13}$ as

$$
\begin{aligned}
\operatorname{prob} & \left\{\left|D_{M}(x, y, z)-\bar{D}(x, y, z)\right|<\frac{k \sigma_{D}(x, y, z)}{\sqrt{M}}\right\} \\
& =\frac{1}{\sqrt{2 \pi}} \int_{-k}^{k} e^{-t^{2} / 2} d t .
\end{aligned}
$$




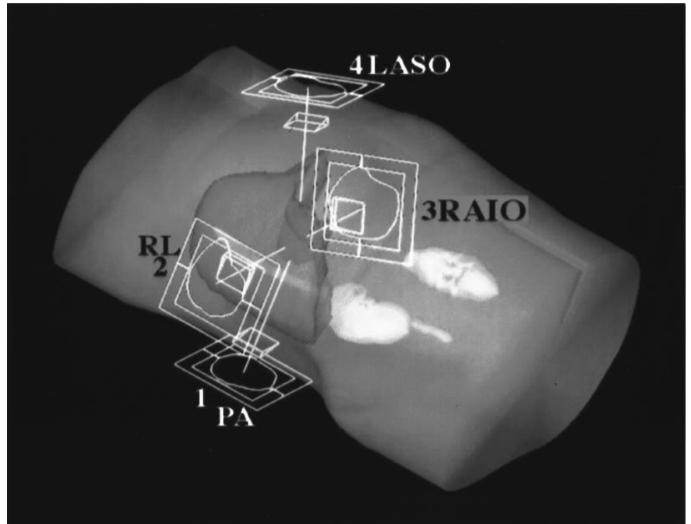

FIG. 2. Treatment planning geometry.

The integral on the right-hand side of Eq. (4) $\approx 0.68$ for $k$ $=1,0.95$ for $k=2$, and 0.98 for $k=3$.

We performed Monte Carlo-based direct simulations ${ }^{11,14-16}$ using the treatment planning system at the University of Michigan (UMPLAN, University of Michigan, Ann Arbor, MI) to confirm the validity of our convolutionbased approach to calculate $\bar{D}(x, y, z)$ and $\sigma_{D}(x, y, z)$. Our procedure is described below.

(1) Randomly sample the setup orientation for each fraction in 3-D from the normal distributions shown in Fig. 1. (The distribution was sampled over $\pm 3 \sigma$ and properly renormalized.)

(2) Recalculate the dose distribution for each fraction in the new geometry using the beam weights (monitor units) from the original configuration (i.e., for a slightly misaligned patient, treatment would proceed under original plan assumptions, but with slightly altered patient geometry).

(3) Combine the dose distribution from each fraction on a common grid to form one possible realization of the course of treatment.

(4) Repeat procedure to calculate many possible realizations of outcome.

(5) Average all realizations to determine distribution of average dose values.

(6) Calculate the distribution of standard deviation values about the average dose values (direct simulation includes effects of fractionation in the calculation of standard deviation).

(7) Compare the dose distribution and standard deviation distribution to the outcome of the convolution calculations.

The treatment planning geometry used for our simulations is shown in Fig. 2. In this problem, the gross tumor volume (GTV) is located in the anterior-inferior portion of the liver. We expanded the PTV from the CTV by an amount equal to the standard deviation of the setup uncertainties in the LR, $\mathrm{AP}$, and SI directions (i.e., LR expansion of $7.4 \mathrm{~mm}=\sigma_{\mathrm{LR}}$, AP expansion of $4.9 \mathrm{~mm}=\sigma_{\mathrm{AP}}$, SI expansion of $5.3 \mathrm{~mm}$ $=\sigma_{\mathrm{SI}}$. . Organ motion due to breathing was not considered for this study. We treated the PTV using a right lateral beam (RL), a posterior-anterior beam (PA), and a wedged pair of oblique beams (RAIO, LASO). The 95\% isodose surface completely covered the PTV (normalized to $100 \%$ at isocenter). Based on the dose to the normal liver (characterized by the effective volume $V_{\text {eff }},{ }^{17}$ the fractional volume of an organ that if uniformly irradiated would result in the same complication probability as the nonuniform irradiated scenario), treatment delivery was planned for 58 fractions at 1.5 gray/fraction delivered twice daily.

We compared the distribution of average dose values calculated using Eq. (1) to the static (initial treatment plan) dose distribution. Also, we calculated dose volume histograms (DVHs) and the effective volume $V_{\text {eff }}$ for the convolved and static treatment plans to determine the gross effects of the setup uncertainties on the treatment plan, as per a liver dose escalation protocol. ${ }^{7-9}$ Next, we calculated the standard deviation of the convolved dose distribution $[\bar{D}(x, y, z)]$ using Eq. (3) and evaluated the result using Eq. (4) to determine the range of potential outcomes in dose about the average dose values.

The results of our convolution-based calculations were also compared to the direct simulations, in the manner described above. We calculated upper and lower bounds on the dose distribution via calculation of $\bar{D}+2 \sigma_{D} / \sqrt{M}$ and $\bar{D}$ $-2 \sigma_{D} / \sqrt{M}$, respectively (95\% confidence limits). We then developed an interpretation of these bounds for the dose distribution and potential applications in the reevaluation of treatment plans using DVHs and $V_{\text {eff }}$ calculations.

\section{RESULTS}

Figure 3(a) shows the RL and PA beam orientation on a single axial CT slice. Figure 3(b) shows a dose difference display, in which the original planning (static) dose distribution is subtracted from the distribution of average dose values $\left(\bar{D}-D_{0}\right)$. Dose to the CTV predicted using a static model is approximately the same as the distribution of average dose values that includes uncertainties, indicating that the margins for the PTV are sufficient. Differences up to $\pm 8 \%$ of the isocenter dose are observed in regions outside the CTV, resulting in a decrease in $V_{\text {eff }}$ for the normal liver sufficient to consider a change in the prescription dose assigned for this treatment plan to maintain a fixed level of toxicity. 9,18

As discussed above, for comparison to the convolutionbased calculations, we performed multiple direct simulations for the treatment plan shown in Fig. 2 (58 fractions per course of treatment at 1.5 gray/fraction). Ten courses of treatment were computed, then averaged and compared to the distribution of average dose values $\bar{D}$ calculated using the convolution method of Eq. (1). A dose difference display (not shown) demonstrated negligible differences $(<0.5 \%)$ in the regions of interest (normal liver, target volumes), with differences observed near the surface of the patient due to artifacts in the convolution calculation arising from discontinuities of the dose distribution at the patient surface. The 

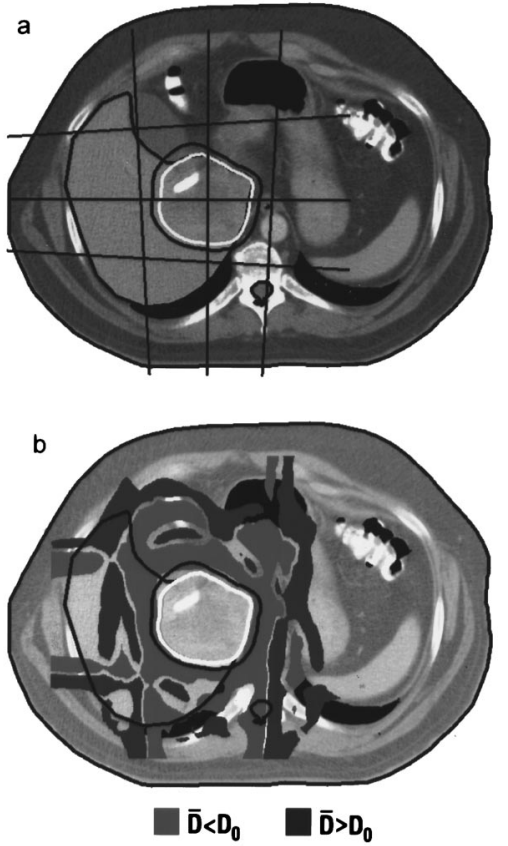

FIG. 3. (a) Single axial slice showing RL and AP beam orientation, (b) dose difference display: $\bar{D}-D_{0}$. The CTV contour is indicated in white. Light gray areas indicate regions where $\bar{D}>D_{0}$, dark gray areas indicate regions where $\bar{D}<D_{0}( \pm 8 \%)$.

DVHs for the target and organs at risk were indistinguishable between the average of the direct simulation calculations and the convolution-based calculation, with $V_{\text {eff }}$ calculations differing by less than $0.2 \%$.

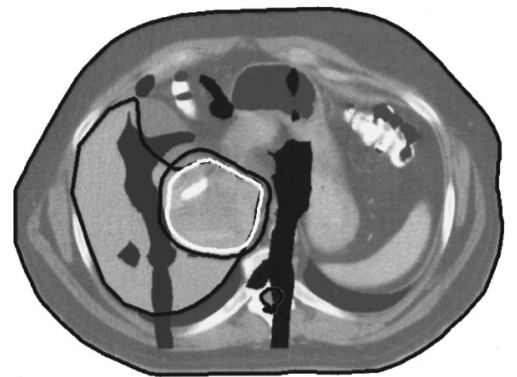

$\overline{\mathbf{D}}<\mathrm{D}_{\mathrm{N}}$

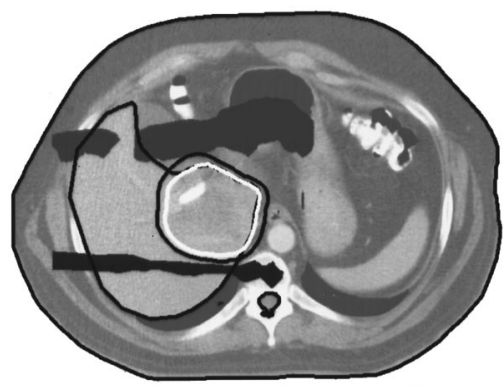

$\overline{\mathrm{D}}>\mathrm{D}_{\mathrm{N}}$

FIG. 4. Dose difference display between two direct simulations of a course of treatment consisting of 56 fractions $D_{N}$ and the distribution of average dose values calculated via convolution $\bar{D}$. Differences up to $\pm 2 \%$ are observed.
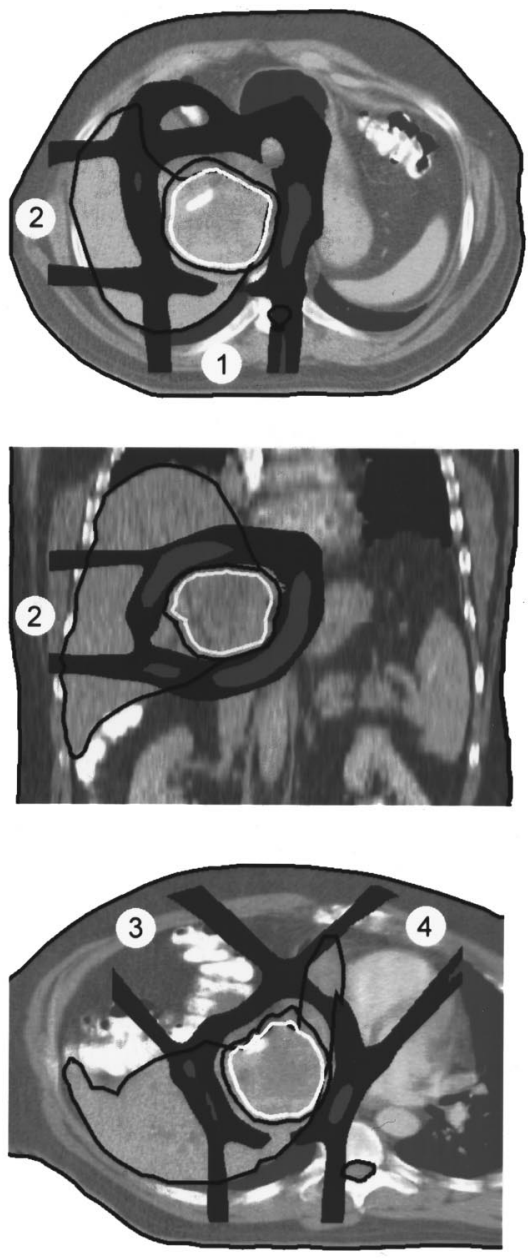

$1-2 \% \square 2-3 \%$

FIG. 5. $\sigma_{D} / \sqrt{M}$ for treatment geometry given in Fig. 2 on an axial, coronal, and oblique $\mathrm{CT}$ reconstruction (beam numbers indicated).

On average, the dose distribution calculated via multiple direct simulations and convolution-based methods [Eq. (1)] agree $(<0.5 \%$ differences $)$, but the dose distribution from any single simulation of a course of treatment $D_{N}$ could deviate from that average [Eq. (1)]. This is shown in Fig. 4 on a single CT slice for the treatment planning geometry in Fig. 2. For this treatment plan, we observed potential differences between direct simulations (58 fractions) and $\bar{D}$ up to $2 \%$ in regions outside the CTV.

Results from calculations of $\sigma_{D}(x, y, z) / \sqrt{M}$ for $M=58$ fractions are shown in Fig. 5 for axial, coronal, and oblique CT reconstructions (in a plane containing beams oblique beams 3 and 4). The spatial distribution of $\sigma_{D} / \sqrt{M}$ computed via Eq. (3) agrees with calculations made via direct simulations (not shown, $<0.2 \%$ differences).

\section{DISCUSSION}

For the example shown, the PTV margins about the CTV were sufficiently large that a realistic dose calculation including random setup uncertainties demonstrated that the static dose calculation correctly predicted the dose to the CTV. 

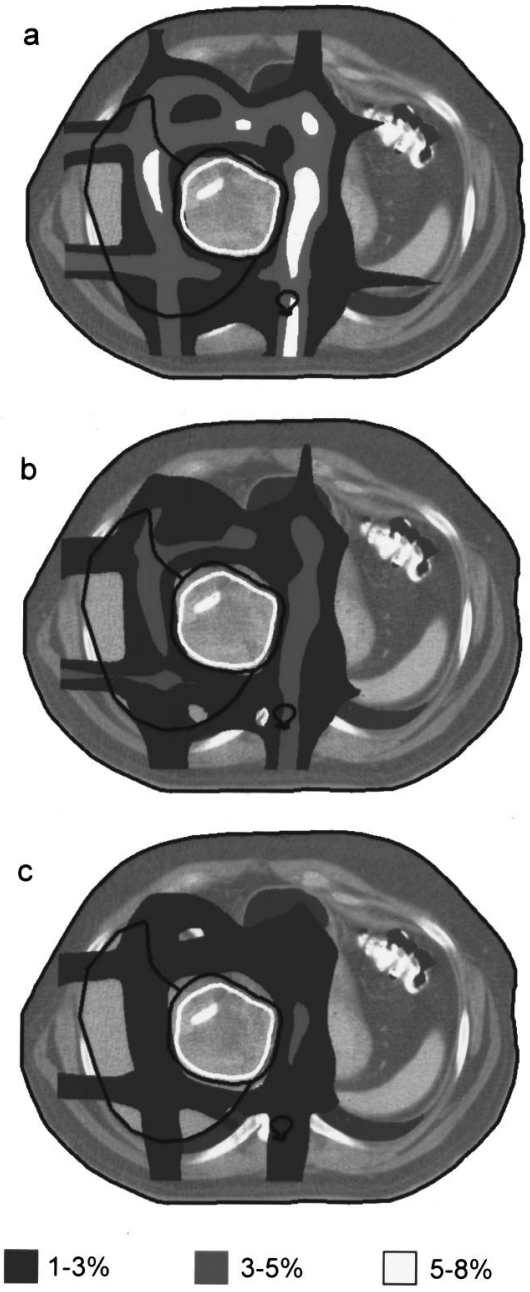

FIG. 6. $\sigma_{D} / \sqrt{M}$ for 10 (a), 20 (b), and 40 fractions (c) on a single axial CT slice.

However, random setup uncertainties during fractionated radiotherapy led to erroneous predictions of the doses to normal tissues. As seen in Fig. 3, the maximum differences between the preplanned and average dose calculation including predicted daily variations are in normal tissue regions corresponding to the beam edges. This result agrees with clinical observations made by Michalski et al. ${ }^{19}$ Though the example shown is specific to a particular treatment planning geometry, it is clear that including the effects of random setup uncertainties in the dose calculations can lead to changes in the prescription dose for protocols based on the predicted dose distribution to normal tissues.

The distribution of average dose values $\bar{D}(x, y, z)$ can be calculated via direct simulations of the treatment, or when appropriate, via a convolution-based calculation applied to the static dose distribution [Eq. (1)]. In both cases, $\bar{D}(x, y, z)$ represents the dose to points $x, y, z$ that would be delivered to an average patient given a very large (infinite) number of fractions. The dose delivered in a finite-fractioned treatment can differ from $\bar{D}$ for even a relatively large number of fractions.

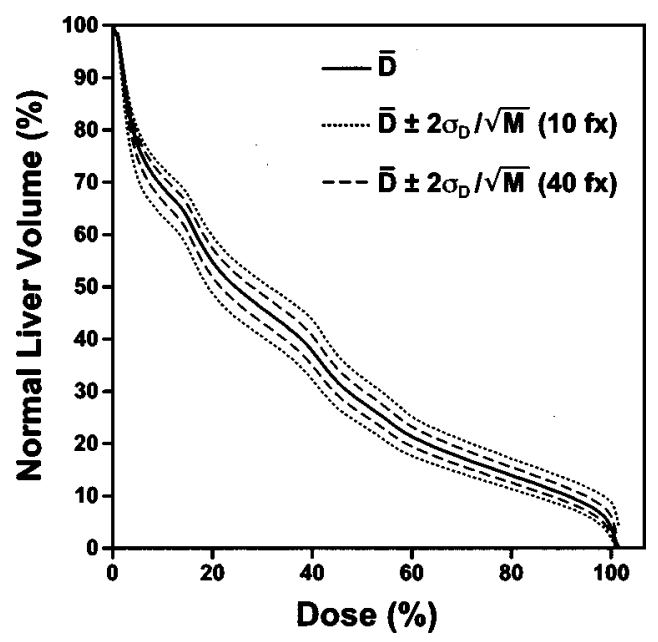

FIG. 7. Cumulative DVH of the normal liver for $\bar{D}$ and $\bar{D} \pm 2\left(\sigma_{D} / \sqrt{M}\right)$ for 10 and 40 fractions.

We have proposed a method to calculate the range of potential outcomes in a real treatment about $\bar{D}(x, y, z)$ using a convolution-based calculation. Using the central limit theorem, this distribution of possible outcomes can be characterized by $\sigma_{D}(x, y, z) / \sqrt{M}$ [where the standard deviation $\sigma_{D}(x, y, z)$ is defined in Eq. (3), and $M$ is the number of fractions in a course of treatment]. Calculation of $\bar{D}(x, y, z)$ $\pm 2 \sigma_{D}(x, y, z) / \sqrt{M}$ can provide population-based confidence limits on our dose distribution (in particular for dose to sensitive structures) and should be considered when assigning the prescription dose.

While the upper and lower bounds on the dose distribution represent true 95\% confidence limits (based on the central limit theorem), the upper bound assumes that all points in the distribution receive a dose greater than the average dose, while the lower bound assumes that all points receive a dose smaller than the average dose. These bounds may not be physically realizable because of conservation of energy (delivered dose) (i.e., if some points in the dose distribution receive a higher than average dose, then other points will receive a lower than average dose). Thus, the upper and lower bounds are true on a voxel-by-voxel basis but do not generally represent the physical (realizable) upper and lower bounds on the total dose distribution. Hence, the spatial distribution of $\sigma_{D}(x, y, z) / \sqrt{M}$ should be considered when evaluating the upper and lower bounds on the average dose distribution $\bar{D}(x, y, z)$.

As the number of fractions in the treatment plan increases, the range of potential outcomes about the average outcome will decrease. This is shown in Fig. 6, where the distribution of $\sigma_{D}(x, y, z) / \sqrt{M}$ is compared for $M=10,20$, and 40 fractions using the planning geometry described in Fig. 2. This is also seen in Fig. 7, which shows cumulative DVHs of the normal liver based on calculations of $\bar{D}$ and $\bar{D} \pm 2 \sigma_{D} / \sqrt{M}$ for 10 and 40 fractions. Clearly, as the number of fractions increases, $\bar{D}$ become a better prediction of the actual dose delivered to the patient.

As noted earlier, the convolution calculation does not 

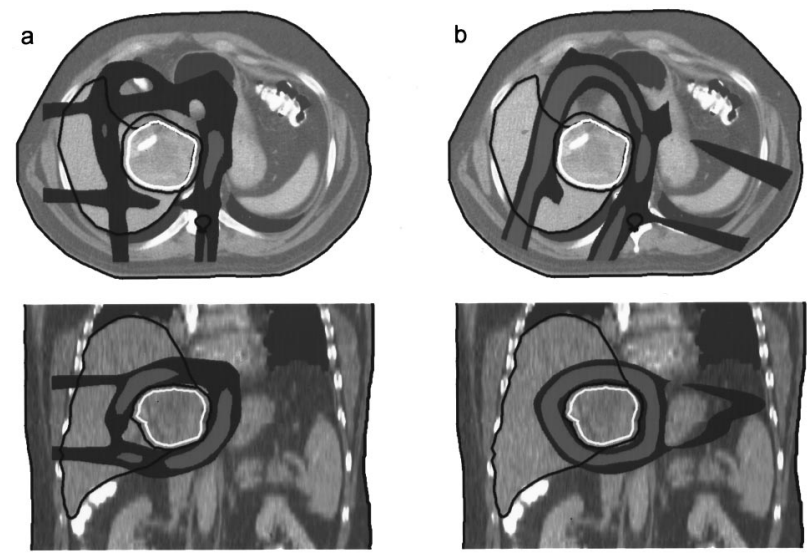

$1-2 \%$

$2-3 \%$

FIG. 8. $\sigma_{D} / \sqrt{M}$ for treatment plan 1 (a) consisting of four beams and treatment plan 2 (b) consisting of three beams.

agree with direct simulations near the patient surface. In general, a convolution-based approach is not valid in regions where the dose distribution itself is not invariant under small changes, for example, near interfaces with large homogeneity differences (lung/normal tissue interface, external surface of the patient). Based on our algorithm (in which the convolution is performed over $\pm 3 \sigma$ ), the average dose distribution calculated via Eq. (1) is not accurate in regions within $3 \sigma$ distance of such an interface, and care must be taken in evaluating the dose to volumes in those regions.

In regions where Eq. (1) can be applied, calculation of $\bar{D}(x, y, z)$ and $\sigma_{D}(x, y, z) / \sqrt{M}$ can be used to evaluate the stability of a treatment plan. For example, beam angles that may lead to unacceptable variations in dose to normal tissues can be adjusted as necessary. Further, setup situations that may require additional efforts (such as daily portal imaging) to minimize setup uncertainties can be identified.

Finally, for a single patient geometry, alternative treatment plans can be evaluated in terms of the effects uncertainties have on the plan. As an example, a second treatment plan was designed for the same patient geometry as Fig. 2. The PTV for the second treatment plan was covered by the 95\% isodose surface using three oblique beams. Treatment delivery was planned using the same fractionation scheme (58 fractions at 1.5 gray/fraction) as plan 1 (Fig. 2). The distribution of $\sigma_{D}(x, y, z) / \sqrt{M}$ was calculated for plan 2 and compared to plan 1 as shown in Fig. 8. The range of possible outcomes about the average expected dose distribution is smaller for treatment plan 1 than for treatment plan 2. Hence, all other factors being equal, we would prefer treatment plan 1 , as we are better able to predict the dose to the patient for plan 1 compared with plan 2.

\section{ACKNOWLEDGMENTS}

Work supported by NIH Grant No. P01-CA59827. Dr. Balter is supported as a Kimmel Scholar.

\footnotetext{
${ }^{a)}$ Electronic mail: aelujan@engin.umich.edu

${ }^{1}$ J. Leong, "Implementation of random positioning error in computerized
}

radiation treatment planning systems as a result of fractionation,' Phys. Med. Biol. 32, 327-334 (1987).

${ }^{2}$ G. J. Kutcher, C. Chui, and T. LoSasso, "'Incorporation of set-up uncertainties in treatment planning calculations,', Int. J. Radiat. Oncol., Biol., Phys. 21, 123 (1991).

${ }^{3}$ C. S. Chui, G. Kutcher, and T. LoSasso, "A convolution method for incorporating uncertainties in dose calculations,' Med. Phys. 19, 814 (1992).

${ }^{4}$ M. A. Hunt, T. E. Schulttheiss, G. Desobry, and G. E. Hanks, ' Convolving setup uncertainties with dose distributions," Med. Phys. 20, 929 (1993).

${ }^{5}$ A. Bel, M. van Herk, and J. M. Lebesque, "Target margins for random geometrical treatment uncertainties in conformal radiotherapy," Med. Phys. 23, 1537-1545 (1996).

${ }^{6}$ A. E. Lujan, E. W. Larsen, J. M. Balter, and R. K. Ten Haken, “A method for incorporating organ motion due to breathing into 3-D dose calculations,', Med. Phys. 26, 715-720 (1999).

${ }^{7}$ T. S. Lawrence, R. J. Tesser, and R. K. Ten Haken, “An application of dose volume histograms to the treatment of intrahepatic malignancies with radiation therapy,' Int. J. Radiat. Oncol., Biol., Phys. 19, 10411047 (1990).

${ }^{8}$ T. S. Lawrence, R. K. Ten Haken, M. L. Kessler, J. M. Robertson, J. T. Lyman, M. L. Lavigne, M. B. Brown, D. J. DuRoss, J. C. Andrews, W. D. Ensminger, and A. S. Lichter, "The use of 3-D dose volume analysis to predict radiation hepatitis,' Int. J. Radiat. Oncol., Biol., Phys. 23, $781-788$ (1992).

${ }^{9}$ C. J. McGinn, R. K. Ten Haken, W. D. Ensminger, S. Walker, S. Wang, and T. S. Lawrence, "The treatment of intraheptatic cancers with radiation doses based on a normal tissue complication probability model,' J. Clin. Oncol. 16, 2246-2252 (1998).

${ }^{10}$ J. E. Schewe, J. M. Balter, K. L. Lam, and R. K. Ten Haken, “Measurement of patient setup errors using port films and a computer-aided graphical alignment tool,' Med. Dosim. 21, 97-104 (1996).

${ }^{11}$ J. H. Killoran, H. M. Kooy, D. J. Gladstone, F. J. Welte, and C. J. Beard, "A numerical simulation of organ motion and daily setup uncertainties: Implications for radiation therapy,' Int. J. Radiat. Oncol., Biol., Phys. 37, 213-221 (1997).

${ }^{12}$ S. Ross, A First Course in Probability, 4th ed. (Macmillian College, New York, 1994).

${ }^{13}$ L. L. Carter and E. D. Caskwell, "Particle Transport Simulation with the Monte Carlo Method,' TID-26607, National Tech. Inf. Service, U.S. Dept. of Commerce, Springfield, VA.

${ }^{14}$ J. C. Stroom, A. G. Visser, J. C. J. de Boer, and H. Huizenga, "Use of coverage probability to quantify results of clinical positioning studies in radiotherapy planning," in Proceedings of the XIth International Conference on the Use of Computers in Radiation Therapy, Manchester, UK, 1994, pp. 264-265.

${ }^{15}$ D. A. Viggars, C. W. Yu, J. S. Lewis, and S. Shalev, “An algorithm for the rapid calculation of the effects of random localization uncertainties on radiotherapy dose distributions,' 'in Proceedings of the XIth International Conference on the Use of Computers in Radiation Therapy, Manchester, UK, 1994, pp. 72-73.

${ }^{16}$ C. D. Mubata, A. M. Bidmead, L. M. Ellingham, and D. P. Dearnaley, "Incorporating patient setup errors in 3D treatment planning," in Proceedings of the XIIth International Conference on the Use of Computers in Radiation Therapy, Salt Lake City, Utah, 1997, pp. 188-190.

${ }^{17}$ G. J. Kutcher and C. Burman, "Calculation of complication probability factors for non-uniform normal tissue irradiation: The effective volume method,' Int. J. Radiat. Oncol., Biol., Phys. 16, 1623-1630 (1989).

${ }^{18}$ R. K. Ten Haken, M. K. Martel, M. L. Kessler, M. B. Hazuka, T. S. Lawrence, J. M. Robertson, A. T. Turrisi, and A. S. Lichter, "Use of $V_{\text {eff }}$ and iso-NTCP in the implementation of dose escalation protocols,' Int. J. Radiat. Oncol., Biol., Phys. 27, 689-695 (1993).

${ }^{19}$ J. M. Michalski, J. W. Wong, R. L. Gerber, D. Yan, A. Cheng, M. V. Graham, M. A. Renna, P. J. Sawyer, and C. A. Perez, "The use of on-line image verification to estimate the variation in radiation therapy dose delivery,', Int. J. Radiat. Oncol., Biol., Phys. 27, 701-716 (1993). 